East Asian Mathematical Journal

Vol. 29 (2013), No. 5, pp. 467-479

http://dx.doi.org/10.7858/eamj.2013.031

\title{
FOURIER-FEYNMAN TRANSFORM AND CONVOLUTION OF FOURIER-TYPE FUNCTIONALS ON WIENER SPACE
}

\author{
Byoung Soo KIM
}

\begin{abstract}
We develop a Fourier-Feynman theory for Fourier-type functionals $\Delta^{k} F$ and $\widehat{\Delta^{k} F}$ on Wiener space. We show that Fourier-Feynman transform and convolution of Fourier-type functionals exist. We also show that the Fourier-Feynman transform of the convolution product of Fourier-type functionals is a product of Fourier-Feynman transforms of each functionals.
\end{abstract}

\section{Introduction and preliminaries}

Let $C_{0}[0, T]$ denote the Wiener space, that is, the space of real valued continuous functions $x$ on $[0, T]$ with $x(0)=0$. Let $\mathcal{M}$ denote the class of all Wiener measurable subsets of $C_{0}[0, T]$ and let $m$ denote Wiener measure. $\left(C_{0}[0, T], \mathcal{M}, m\right)$ is a complete measure space and we denote the Wiener integral of a functional $F$ by

$$
\int_{C_{0}[0, T]} F(x) d m(x)
$$

A subset $E$ of $C_{0}[0, T]$ is said to be scale-invariant measurable provided $\rho E$ is Wiener measurable for every $\rho>0$, and a scale-invariant measurable set $N$ is said to be scale-invariant null provided $m(\rho N)=0$ for every $\rho>0$. A property that holds except on a scale-invariant null set is said to hold scaleinvariant almost everywhere (s-a.e.). Given two complex-valued functions $F$ and $G$ on $C_{0}[0, T]$, we say that $F=G$ s-a.e. if $F(\rho x)=G(\rho x)$ for $m$ almost every $x \in C_{0}[0, T]$ for all $\rho>0$.

Let $\mathbb{C}_{+}$and $\mathbb{C}_{+}^{\sim}$ denote the sets of complex numbers with positive real part and nonzero complex numbers with nonnegative real part, respectively.

Received January 24, 2013; Accepted August 14, 2013

2000 Mathematics Subject Classification. 28C20, 60J65.

Key words and phrases. Wiener space, Feynman integral, Fourier-Feynman transform, convolution, Fourier-type functional.

This study was partially supported by Seoul National University of Science and Technology. 
Let $F$ be a complex valued scale-invariant measurable functional on $C_{0}[0, T]$ such that

$$
J(\lambda)=\int_{C_{0}[0, T]} F\left(\lambda^{-1 / 2} x\right) d m(x)
$$

exists as a finite number for all real $\lambda>0$. If there exists an analytic function $J^{*}(\lambda)$ on $\mathbb{C}_{+}$such that $J^{*}(\lambda)=J(\lambda)$ for all $\lambda>0$, then $J^{*}(\lambda)$ is defined to be the analytic Wiener integral of $F$ over $C_{0}[0, T]$ with parameter $\lambda$, and for $\lambda \in \mathbb{C}_{+}$we write

$$
\int_{C_{0}[0, T]}^{\mathrm{anw}_{\lambda}} F(x) d m(x)=J^{*}(\lambda) .
$$

Let $F$ be a functional on $C_{0}[0, T]$ such that $\int_{C_{0}[0, T]}^{a_{\lambda} w_{\lambda}} F(x) d m(x)$ exists for all $\lambda \in \mathbb{C}_{+}$. If the following limit exists for nonzero real number $q$, then we call it the analytic Feynman integral of $F$ over $C_{0}[0, T]$ with parameter $q$ and we write

$$
\int_{C_{0}[0, T]}^{\operatorname{anf}_{q}} F(x) d m(x)=\lim _{\lambda \rightarrow-i q} \int_{C_{0}[0, T]}^{\mathrm{anw}_{\lambda}} F(x) d m(x)
$$

where $\lambda \rightarrow-i q$ through $\mathbb{C}_{+}$.

Now we introduce the definitions of analytic Fourier-Feynman transform and convolution product for functionals defined on $C_{0}[0, T]$. Let $1 \leq p<\infty$ and let $q$ be a nonzero real number.

Definition 1. Let $F$ be a functional on $C_{0}[0, T]$. For $\lambda \in \mathbb{C}_{+}$and $y \in C_{0}[0, T]$, let

$$
T_{\lambda}(F)(y)=\int_{C_{0}[0, T]}^{\mathrm{anw}_{\lambda}} F(x+y) d m(x) .
$$

For $1<p<\infty$, we define the $L_{p}$ analytic Fourier-Feynman transform $T_{q}^{(p)}(F)$ of $F$ on $C_{0}[0, T]$ by the formula $\left(\lambda \in \mathbb{C}_{+}\right)$

$$
T_{q}^{(p)}(F)(y)=\underset{\lambda \rightarrow-i q}{\lim . T_{\lambda}} T_{\lambda}(F)(y)
$$

whenever this limit exists; that is, for each $\rho>0$,

$$
\lim _{\lambda \rightarrow-i q} \int_{C_{0}[0, T]}\left|T_{\lambda}(F)(\rho x)-T_{q}^{(p)}(F)(\rho x)\right|^{p^{\prime}} d m(x)=0
$$

where $1 / p+1 / p^{\prime}=1$. We define the $L_{1}$ analytic Fourier-Feynman transform $T_{q}^{(1)}(F)$ of $F$ by $\left(\lambda \in \mathbb{C}_{+}\right)$

$$
T_{q}^{(1)}(F)(y)=\lim _{\lambda \rightarrow-i q} T_{\lambda}(F)(y),
$$

for $s$-a.e. $y \in C_{0}[0, T]$, whenever this limit exists $[2,7,8,9]$.

By the definition of the analytic Feynman integral and the $L_{1}$ analytic Fourier-Feynman transform, it is easy to see that for a nonzero real number $q$,

$$
T_{q}^{(1)}(F)(y)=\int_{C_{0}[0, T]}^{\operatorname{anf}_{q}} F(x+y) d m(x)
$$


and

$$
T_{q}^{(1)}(F)(0)=\int_{C_{0}[0, T]}^{\operatorname{anf}_{q}} F(x) d m(x) .
$$

Definition 2. Let $F$ and $G$ be functionals on $C_{0}[0, T]$. For $\lambda \in \mathbb{C}_{+}$and $y \in C_{0}[0, T]$, we define their convolution product by

$$
(F * G)_{\lambda}(y)=\int_{C_{0}[0, T]}^{\mathrm{anw}_{\lambda}} F\left(\frac{y+x}{\sqrt{2}}\right) G\left(\frac{y-x}{\sqrt{2}}\right) d m(x) .
$$

Moreover if $\lambda=-i q$ for nonzero real $q$, the convolution product is defined by

$$
(F * G)_{q}(y)=\int_{C_{0}[0, T]}^{\operatorname{anf}_{q}} F\left(\frac{y+x}{\sqrt{2}}\right) G\left(\frac{y-x}{\sqrt{2}}\right) d m(x)
$$

if it exists $[7,8,14,15]$.

It is easy to see that commutative law holds for the convolution product [7].

Various results involving Fourier-Feynman transform on Wiener space have been established and research based on this definition is continuing at the present time [1, 3, 4, 5, 12]. Recently, Kim, Kim and Yang extended the concepts of Fourier-Feynman transform and convolution on Wiener space to the concept of Fourier-Yeh-Feynman transform and convolution on Yeh-Wiener space $[10,11]$. For a detailed survey of the previous work on the FourierFeynman transfprm and related topics, see [13].

Now we describe the class of functionals that we work with in this paper. Recently, Chung and Tuan [6] introduced the Fourier-type functionals via the Fourier transform on Wiener space and investigate some properties of the Fourier-type functionals.

Let $\hat{f}$ be the Fourier transform of $f$,

$$
\hat{f}(\vec{\xi})=\left(\frac{1}{2 \pi}\right)^{n / 2} \int_{\mathbb{R}^{n}} f(\vec{u}) \exp \{i \vec{u} \cdot \vec{\xi}\} d \vec{u}, \quad \vec{\xi} \in \mathbb{R}^{n}
$$

where $\vec{u} \cdot \vec{\xi}=u_{1} \xi_{1}+\cdots+u_{n} \xi_{n}$.

Let $\mathcal{S}\left(\mathbb{R}^{n}\right)$ be the Schwartz space of infinitely differentiable functions $f(\vec{u})$ decaying at infinity together with all its derivatives faster than any polynomial of $|\vec{u}|^{-1}$. Note that the Fourier transform is an isomorphism on the Schwartz space $\mathcal{S}\left(\mathbb{R}^{n}\right)$. Also, $\Delta^{k} f$ and $\widehat{\Delta^{k} f}$ are elements of $\mathcal{S}\left(\mathbb{R}^{n}\right)$ for all $k=0,1,2, \ldots$, where $\Delta$ denotes the Laplacian

$$
\Delta=\frac{\partial^{2}}{\partial u_{1}^{2}}+\frac{\partial^{2}}{\partial u_{2}^{2}}+\cdots+\frac{\partial^{2}}{\partial u_{n}^{2}} .
$$

Note that $\mathcal{S}\left(\mathbb{R}^{n}\right)$ is a subset of $L^{1}\left(\mathbb{R}^{n}\right)$, more precisely, for $\phi \in \mathcal{S}\left(\mathbb{R}^{n}\right)$ we know that

$$
\begin{aligned}
\|\phi\|_{1} & =\int_{\mathbb{R}^{n}}\left(1+|\vec{u}|^{2}\right)^{-1}\left(1+|\vec{u}|^{2}\right)|\phi(\vec{u})| d \vec{u} \\
& \leq \pi^{n}\left[\|\phi\|_{\infty}+\left\||\cdot|^{2} \phi(\cdot)\right\|_{\infty}\right]<\infty .
\end{aligned}
$$


Now we introduce the Fourier-type functionals defined on Wiener space.

Definition 3. Let $\left\{\alpha_{1}, \alpha_{2}, \ldots, \alpha_{n}\right\}$ be an orthonormal set of functions in $L_{2}[0, T]$ and let $k$ be a nonnegative integer. For $f \in \mathcal{S}\left(\mathbb{R}^{n}\right)$, the Fourier-type functionals $\Delta^{k} F$ and $\widehat{\Delta^{k} F}$ on Wiener space $C_{0}[0, T]$ are defined by

$$
\Delta^{k} F(x)=\Delta^{k} f(\langle\vec{\alpha}, x\rangle)
$$

and

$$
\widehat{\Delta^{k} F}(x)=\widehat{\Delta^{k}} f(\langle\vec{\alpha}, x\rangle)
$$

where $\langle\vec{\alpha}, x\rangle=\left(\left\langle\alpha_{1}, x\right\rangle, \ldots,\left\langle\alpha_{n}, x\right\rangle\right)$ and $\left\langle\alpha_{j}, x\right\rangle$ denotes the Paley-WienerZigmund stochastic integral for $j=1,2, \ldots, n$.

In this paper, we develop a Fourier-Feynman theory for Fourier-type functionals $\Delta^{k} F$ and $\widehat{\Delta^{k} F}$ on Wiener space. In Section 2, we show that FourierFeynman transform of Fourier-type functionals exists. In Section 3, we show that convolution of Fourier-type functionals exists. We also show that the Fourier-Feynman transform of the convolution product of Fourier-type functionals is a product of Fourier-Feynman transforms of each functionals.

We close this section by introducing a well-known Wiener integration formula for functionals $f(\langle\vec{\alpha}, x\rangle)=f\left(\left\langle\alpha_{1}, x\right\rangle, \ldots,\left\langle\alpha_{n}, x\right\rangle\right)$;

$$
\int_{C_{0}[0, T]} f(\langle\vec{\alpha}, x\rangle) d m(x)=(2 \pi)^{-n / 2} \int_{\mathbb{R}^{n}} f(\vec{u}) \exp \left\{-\frac{1}{2}|\vec{u}|^{2}\right\} d \vec{u}
$$

where $|\vec{u}|^{2}=\sum_{j=1}^{n} u_{j}^{2}$.

\section{Fourier-Feynman transform of Fourier-type functionals}

In this section we show that the $L_{p}$ analytic Fourier-Feynman transforms $T_{q}^{(p)}\left(\Delta^{k} F\right)$ and $T_{q}^{(p)}\left(\widehat{\Delta^{k} F}\right)$ of the Fourier-type functionals exist. We also establish a relationship between $T_{q}^{(p)}\left(\Delta^{k} F\right)$ and $T_{q}^{(p)}\left(\widehat{\Delta^{k} F}\right)$.

Theorem 2.1. Let the Fourier-type functional $\Delta^{k} F$ be given by (9). Then for all $p$ with $1 \leq p<\infty$ and for all nonzero real number $q$, the $L_{p}$ analytic Fourier-Feynman transform $T_{q}^{(p)}\left(\Delta^{k} F\right)$ exists and is given by the formula

$$
T_{q}^{(p)}\left(\Delta^{k} F\right)(y)=\left(\frac{-i q}{2 \pi}\right)^{n / 2} \int_{\mathbb{R}^{n}} \Delta^{k} f(\vec{u}) \exp \left\{\frac{i q}{2}|\vec{u}-\langle\vec{\alpha}, y\rangle|^{2}\right\} d \vec{u}
$$

for s-a.e. $y \in C_{0}[0, T]$.

Proof. Using the Wiener integration formula (11), we obtain

$$
\begin{aligned}
T_{\lambda}\left(\Delta^{k} F\right)(y) & =\int_{C_{0}[0, T]} \Delta^{k} f\left(\lambda^{-1 / 2}\langle\vec{\alpha}, x\rangle+\langle\vec{\alpha}, y\rangle\right) d m(x) \\
& =\left(\frac{\lambda}{2 \pi}\right)^{n / 2} \int_{\mathbb{R}^{n}} \Delta^{k} f(\vec{u}+\langle\vec{\alpha}, y\rangle) \exp \left\{-\frac{\lambda}{2}|\vec{u}|^{2}\right\} d \vec{u} \\
& =\left(\frac{\lambda}{2 \pi}\right)^{n / 2} \int_{\mathbb{R}^{n}} \Delta^{k} f(\vec{u}) \exp \left\{-\frac{\lambda}{2}|\vec{u}-\langle\vec{\alpha}, y\rangle|^{2}\right\} d \vec{u}
\end{aligned}
$$


for all $\lambda>0$ and $y \in C_{0}[0, T]$. Let $\lambda \in \mathbb{C}_{+}^{\sim}$ and let $\left\{\lambda_{n}\right\}$ be a sequence in $\mathbb{C}_{+}^{\sim}$ which converges to $\lambda$. Since $\Delta^{k} f$ is an element of $\mathcal{S}\left(\mathbb{R}^{n}\right)$ and since $\mathcal{S}\left(\mathbb{R}^{n}\right)$ is a subset of $L^{1}\left(\mathbb{R}^{n}\right)$, we can apply dominated convergence theorem to show that

$$
\lim _{n \rightarrow \infty} T_{\lambda_{n}}\left(\Delta^{k} F\right)(y)=T_{\lambda}\left(\Delta^{k} F\right)(y)
$$

and so $T_{\lambda}\left(\Delta^{k} F\right)(y)$ is a continuous function of $\lambda$ in $\mathbb{C}_{+}^{\sim}$. Let $D$ be a closed contour in $\mathbb{C}_{+}$. By the Fubini theorem and the Cauchy theorem,

$$
\int_{D} \int_{\mathbb{R}^{n}} \Delta^{k} f(\vec{u}) \exp \left\{-\frac{\lambda}{2}|\vec{u}-\langle\vec{\alpha}, y\rangle|^{2}\right\} d \vec{u} d \lambda=0 .
$$

Hence by the Morera's theorem, $\int_{\mathbb{R}^{n}} \Delta^{k} f(\vec{u}) \exp \left\{-\frac{\lambda}{2}|\vec{u}-\langle\vec{\alpha}, y\rangle|^{2}\right\} d \vec{u}$ is an analytic function of $\lambda$ in $\mathbb{C}_{+}$. Hence for $\lambda \in \mathbb{C}_{+}$and $y \in C_{0}[0, T]$,

$$
T_{\lambda}\left(\Delta^{k} F\right)(y)=\left(\frac{\lambda}{2 \pi}\right)^{n / 2} \int_{\mathbb{R}^{n}} \Delta^{k} f(\vec{u}) \exp \left\{-\frac{\lambda}{2}|\vec{u}-\langle\vec{\alpha}, y\rangle|^{2}\right\} d \vec{u} .
$$

In case $p=1$, by the dominated convergence theorem,

$$
\begin{aligned}
T_{q}^{(1)}\left(\Delta^{k} F\right)(y) & =\lim _{\lambda \rightarrow-i q} T_{\lambda}\left(\Delta^{k} F\right)(y) \\
& =\left(\frac{-i q}{2 \pi}\right)^{n / 2} \int_{\mathbb{R}^{n}} \Delta^{k} f(\vec{u}) \exp \left\{\frac{i q}{2}|\vec{u}-\langle\vec{\alpha}, y\rangle|^{2}\right\} d \vec{u}
\end{aligned}
$$

for $y \in C_{0}[0, T]$. If $1<p<\infty$, again by the dominated convergence theorem, the Wiener integral

$\int_{C_{0}[0, T]}\left|\left(\frac{-i q}{2 \pi}\right)^{n / 2} \int_{\mathbb{R}^{n}} \Delta^{k} f(\vec{u}) \exp \left\{\frac{i q}{2}|\vec{u}-\langle\vec{\alpha}, \rho y\rangle|^{2}\right\} d \vec{u}-T_{\lambda}\left(\Delta^{k} F\right)(\rho y)\right|^{p^{\prime}} d m(y)$ goes to 0 as $\lambda \rightarrow-i q$ for each $\rho>0$. Hence $T_{q}^{(p)}\left(\Delta^{k} F\right)(y)$ exists and is given by (12) for s-a.e. $y \in C_{0}[0, T]$ and for all desired values of $p$ and $q$.

As we have seen in (5), the $L_{1}$ analytic Fourier-Feynman transform of $F$ evaluated at 0 is equal to the analytic Feynman integral of $F$. Hence we have the following corollary.

Corollary 2.2. Let the Fourier-type functional $\Delta^{k} F$ be given by (9). Then $\Delta^{k} F$ is analytic Wiener integrable and

$$
\int_{C_{0}[0, T]}^{\mathrm{anw}_{\lambda}} \Delta^{k} F(x) d m(x)=\left(\frac{\lambda}{2 \pi}\right)^{n / 2} \int_{\mathbb{R}^{n}} \Delta^{k} f(\vec{u}) \exp \left\{-\frac{\lambda}{2}|\vec{u}|^{2}\right\} d \vec{u}
$$

for all $\lambda \in \mathbb{C}_{+}$. Moreover, $\Delta^{k} F$ is analytic Feynman integrable and

$$
\int_{C_{0}[0, T]}^{\operatorname{anf}_{q}} \Delta^{k} F(x) d m(x)=\left(\frac{-i q}{2 \pi}\right)^{n / 2} \int_{\mathbb{R}^{n}} \Delta^{k} f(\vec{u}) \exp \left\{\frac{i q}{2}|\vec{u}|^{2}\right\} d \vec{u}
$$

for all nonzero real number $q$.

In the following theorem we evaluate Fourier-Feynman transform of $\widehat{\Delta^{k} F}$. 
Theorem 2.3. Let the Fourier-type functional $\widehat{\Delta^{k} F}$ be given by (10). Then for all $p$ with $1 \leq p<\infty$ and for all nonzero real number $q$, the $L_{p}$ analytic Fourier-Feynman transform $T_{q}^{(p)}\left(\widehat{\Delta^{k} F}\right)$ exists and is given by the formula

$$
T_{q}^{(p)}\left(\widehat{\Delta^{k} F}\right)(y)=\left(\frac{1}{2 \pi}\right)^{n / 2} \int_{\mathbb{R}^{n}} \Delta^{k} f(\vec{u}) \exp \left\{-\frac{i}{2 q}|\vec{u}|^{2}+i\langle\vec{\alpha}, y\rangle \cdot \vec{u}\right\} d \vec{u}
$$

for $s$-a.e. $y \in C_{0}[0, T]$.

Proof. Using the Wiener integration formula (11) we obtain

$$
\begin{aligned}
T_{\lambda}\left(\widehat{\Delta^{k} F}\right)(y) & =\int_{C_{0}[0, T]} \widehat{\Delta^{k}} f\left(\lambda^{-1 / 2}\langle\vec{\alpha}, x\rangle+\langle\vec{\alpha}, y\rangle\right) d m(x) \\
& =\left(\frac{\lambda}{2 \pi}\right)^{n / 2} \int_{\mathbb{R}^{n}} \widehat{\Delta^{k}} f(\vec{v}+\langle\vec{\alpha}, y\rangle) \exp \left\{-\frac{\lambda}{2}|\vec{v}|^{2}\right\} d \vec{v} \\
& =\left(\frac{\lambda}{2 \pi}\right)^{n / 2} \int_{\mathbb{R}^{n}} \widehat{\Delta^{k}} f(\vec{v}) \exp \left\{-\frac{\lambda}{2}|\vec{v}-\langle\vec{\alpha}, y\rangle|^{2}\right\} d \vec{v}
\end{aligned}
$$

for all $\lambda>0$ and $y \in C_{0}[0, T]$. By (8) we have

$$
\begin{aligned}
& T_{\lambda}\left(\widehat{\Delta^{k} F}\right)(y) \\
= & \left(\frac{\lambda}{2 \pi}\right)^{n / 2}\left(\frac{1}{2 \pi}\right)^{n / 2} \int_{\mathbb{R}^{n}} \int_{\mathbb{R}^{n}} \Delta^{k} f(\vec{u}) \exp \left\{-\frac{\lambda}{2}|\vec{v}-\langle\vec{\alpha}, y\rangle|^{2}+i \vec{u} \cdot \vec{v}\right\} d \vec{u} d \vec{v} .
\end{aligned}
$$

But since

$$
\begin{aligned}
& \int_{\mathbb{R}^{n}} \exp \left\{-\frac{\lambda}{2}|\vec{v}-\langle\vec{\alpha}, y\rangle|^{2}+i \vec{u} \cdot \vec{v}\right\} d \vec{v} \\
= & \int_{\mathbb{R}^{n}} \exp \left\{-\frac{\lambda}{2}\left|\vec{v}-\left[\langle\vec{\alpha}, y\rangle+\frac{i}{\lambda} \vec{u}\right]\right|^{2}+i\langle\vec{\alpha}, y\rangle \cdot \vec{u}-\frac{1}{2 \lambda}|\vec{u}|^{2}\right\} d \vec{v} \\
= & \left(\frac{2 \pi}{\lambda}\right)^{n / 2} \exp \left\{-\frac{1}{2 \lambda}|\vec{u}|^{2}+i\langle\vec{\alpha}, y\rangle \cdot \vec{u}\right\},
\end{aligned}
$$

we have

$$
T_{\lambda}\left(\widehat{\Delta^{k} F}\right)(y)=\left(\frac{1}{2 \pi}\right)^{n / 2} \int_{\mathbb{R}^{n}} \Delta^{k} f(\vec{u}) \exp \left\{-\frac{1}{2 \lambda}|\vec{u}|^{2}+i\langle\vec{\alpha}, y\rangle \cdot \vec{u}\right\} d \vec{u}
$$

for all $\lambda>0$ and $y \in C_{0}[0, T]$. Let $\lambda \in \mathbb{C}_{+}^{\sim}$ and let $\left\{\lambda_{n}\right\}$ be a sequence in $\mathbb{C}_{+}^{\sim}$ which converges to $\lambda$. Since $\Delta^{k} f$ is integrable on $\mathbb{R}^{n}$, we can apply dominated convergence theorem to show that

$$
\lim _{n \rightarrow \infty} T_{\lambda_{n}}\left(\widehat{\Delta^{k} F}\right)(y)=T_{\lambda}\left(\widehat{\Delta^{k} F}\right)(y)
$$

and so $T_{\lambda}\left(\widehat{\Delta^{k} F}\right)(y)$ is a continuous function of $\lambda$ in $\mathbb{C}_{+}^{\sim}$. Let $D$ be a closed contour in $\mathbb{C}_{+}$. By the Fubini theorem and the Cauchy theorem,

$$
\int_{D} \int_{\mathbb{R}^{n}} \Delta^{k} f(\vec{u}) \exp \left\{-\frac{1}{2 \lambda}|\vec{u}|^{2}+i\langle\vec{\alpha}, y\rangle \cdot \vec{u}\right\} d \vec{u} d \lambda=0
$$


Hence by the Morera's theorem, $\int_{\mathbb{R}^{n}} \Delta^{k} f(\vec{u}) \exp \left\{-\frac{1}{2 \lambda}|\vec{u}|^{2}+i\langle\vec{\alpha}, y\rangle \cdot \vec{u}\right\} d \vec{u}$ is an analytic function of $\lambda$ in $\mathbb{C}_{+}$. Hence for $\lambda \in \mathbb{C}_{+}$and $y \in C_{0}[0, T]$,

$$
T_{\lambda}\left(\widehat{\Delta^{k} F}\right)(y)=\left(\frac{1}{2 \pi}\right)^{n / 2} \int_{\mathbb{R}^{n}} \Delta^{k} f(\vec{u}) \exp \left\{-\frac{1}{2 \lambda}|\vec{u}|^{2}+i\langle\vec{\alpha}, y\rangle \cdot \vec{u}\right\} d \vec{u} .
$$

In case $p=1$, by the dominated convergence theorem,

$$
\begin{aligned}
T_{q}^{(1)}\left(\widehat{\Delta^{k} F}\right)(y) & =\lim _{\lambda \rightarrow-i q} T_{\lambda}\left(\widehat{\Delta^{k} F}\right)(y) \\
& =\left(\frac{1}{2 \pi}\right)^{n / 2} \int_{\mathbb{R}^{n}} \Delta^{k} f(\vec{u}) \exp \left\{-\frac{i}{2 q}|\vec{u}|^{2}+i\langle\vec{\alpha}, y\rangle \cdot \vec{u}\right\} d \vec{u}
\end{aligned}
$$

for $y \in C_{0}[0, T]$. If $1<p<\infty$, again by the dominated convergence theorem, the Wiener integral

$$
\begin{aligned}
\int_{C_{0}[0, T]} \mid & \left(\frac{1}{2 \pi}\right)^{n / 2} \int_{\mathbb{R}^{n}} \Delta^{k} f(\vec{u}) \exp \left\{-\frac{i}{2 q}|\vec{u}|^{2}+\left.i\langle\vec{\alpha}, \rho y\rangle \cdot \vec{u}\right|^{2}\right\} d \vec{u} \\
& -\left.T_{\lambda}\left(\widehat{\Delta^{k} F}\right)(\rho y)\right|^{p^{\prime}} d m(y)
\end{aligned}
$$

goes to 0 as $\lambda \rightarrow-i q$ for each $\rho>0$. Hence $T_{q}^{(p)}\left(\widehat{\Delta^{k} F}\right)(y)$ exists and is given by (15) for $s$-a.e. $y \in C_{0}[0, T]$ and for all desired values of $p$ and $q$.

The following corollary is a parallel result of Corollary 2.2 for the Fouriertype functional $\widehat{\Delta^{k} F}$. It can be obtained by (15) and (5).

Corollary 2.4. Let the Fourier-type functional $\widehat{\Delta^{k} F}$ be given by (10). Then $\widehat{\Delta^{k} F}$ is analytic Wiener integrable and

$$
\int_{C_{0}[0, T]}^{\mathrm{anw}_{\lambda}} \widehat{\Delta^{k} F}(x) d m(x)=\left(\frac{1}{2 \pi}\right)^{n / 2} \int_{\mathbb{R}^{n}} \Delta^{k} f(\vec{v}) \exp \left\{-\frac{1}{2 \lambda}|\vec{v}|^{2}\right\} d \vec{v}
$$

for all $\lambda \in \mathbb{C}_{+}$. Moreover, $\widehat{\Delta^{k} F}$ is analytic Feynman integrable and

$$
\int_{C_{0}[0, T]}^{\operatorname{anf}_{q}} \widehat{\Delta^{k} F}(x) d m(x)=\left(\frac{1}{2 \pi}\right)^{n / 2} \int_{\mathbb{R}^{n}} \Delta^{k} f(\vec{u}) \exp \left\{-\frac{i}{2 q}|\vec{u}|^{2}\right\} d \vec{u}
$$

for any nonzero real number $q$.

Comparing equations (12) and (15), we obtain a relationship between the Fourier-Feynman transforms of $\Delta^{k} F$ and $\widehat{\Delta^{k} F}$ as in the following theorem.

Theorem 2.5. Let the Fourier-type functionals $\Delta^{k} F$ and $\widehat{\Delta^{k} F}$ be given by (9) and (10), respectively. Let $1 \leq p<\infty$ and let $q$ be any nonzero real number. Then we have

$$
T_{q}^{(p)}\left(\widehat{\Delta^{k} F}\right)(y)=(-i q)^{n / 2} T_{-1 / q}^{(p)}\left(\Delta^{k} F\right)(q y) \exp \left\{\frac{i q}{2}|\langle\vec{\alpha}, y\rangle|^{2}\right\}
$$

for $s$-a.e. $y \in C_{0}[0, T]$. 
Proof. Replacing $q$ with $-1 / q$ and $y$ with $q y$ in (12), we have

$$
\begin{aligned}
& T_{-1 / q}^{(p)}\left(\Delta^{k} F\right)(q y) \\
= & \left(\frac{i}{2 \pi q}\right)^{n / 2} \int_{\mathbb{R}^{n}} \Delta^{k} f(\vec{u}) \exp \left\{-\frac{i}{2 q}|\vec{u}-q\langle\vec{\alpha}, y\rangle|^{2}\right\} d \vec{u} \\
= & \left(\frac{i}{2 \pi q}\right)^{n / 2} \int_{\mathbb{R}^{n}} \Delta^{k} f(\vec{u}) \exp \left\{-\frac{i}{2 q}|\vec{u}|^{2}+i\langle\vec{\alpha}, y\rangle \cdot \vec{u}-\frac{i q}{2}|\langle\vec{\alpha}, y\rangle|^{2}\right\} d \vec{u} .
\end{aligned}
$$

Finally by (15) we have the desired result.

If we take $y=0$ in (18) above, by (5) we have a relationship between the analytic Feynman integrals of $\Delta^{k} F$ and $\widehat{\Delta^{k} F}$ as follows.

Corollary 2.6. Let the Fourier-type functionals $\Delta^{k} F$ and $\widehat{\Delta^{k} F}$ be given by (9) and (10), respectively. Then we have

$$
\int_{C_{0}[0, T]}^{\operatorname{anf}_{q}} \widehat{\Delta^{k} F}(x) d m(x)=(-i q)^{n / 2} \int_{C_{0}[0, T]}^{\mathrm{anf}_{-1 / q}} \Delta^{k} F(x) d m(x)
$$

for any nonzero real number $q$.

\section{Convolution of Fourier-type functionals}

In this section we will show the existence of the convolution product for the Fourier-type functionals on Wiener space. We also show that the FourierFeynman transform of the convolution product of Fourier-type functionals is a product of Fourier-Feynman transforms of each functionals.

Lemma 3.1. Let $f, g \in \mathcal{S}\left(\mathbb{R}^{n}\right)$ and let $k$ be a nonnegative integer. For $\lambda \in \mathbb{C}_{+}^{\sim}$ and $\vec{w} \in \mathbb{R}^{n}$, let

$$
h(\lambda, \vec{w})=\left(\frac{\lambda}{2 \pi}\right)^{n / 2} \int_{\mathbb{R}^{n}} \Delta^{k} f\left(\frac{\vec{w}+\vec{u}}{\sqrt{2}}\right) \Delta^{k} g\left(\frac{\vec{w}-\vec{u}}{\sqrt{2}}\right) \exp \left\{-\frac{\lambda}{2}|\vec{u}|^{2}\right\} d \vec{u} .
$$

Then $h(\lambda,\langle\vec{\alpha}, y\rangle)$ exists for a.e. $y \in C_{0}[0, T]$.

Proof. First note that

$$
\int_{\mathbb{R}^{n}}|h(\lambda, \vec{w})| d \vec{w} \leq\left(\frac{|\lambda|}{2 \pi}\right)^{n / 2} \int_{\mathbb{R}^{n}} \int_{\mathbb{R}^{n}}\left|\Delta^{k} f\left(\frac{\vec{w}+\vec{u}}{\sqrt{2}}\right) \Delta^{k} g\left(\frac{\vec{w}-\vec{u}}{\sqrt{2}}\right)\right| d \vec{u} d \vec{w} .
$$

Letting $\vec{v}=(\vec{w}+\vec{u}) / \sqrt{2}$ and $\vec{r}=(\vec{w}-\vec{u}) / \sqrt{2}$, we have

$$
\begin{aligned}
\int_{\mathbb{R}^{n}}|h(\lambda, \vec{w})| d \vec{w} & \leq\left(\frac{|\lambda|}{2 \pi}\right)^{n / 2} \int_{\mathbb{R}^{n}} \int_{\mathbb{R}^{n}}\left|\Delta^{k} f(\vec{v}) \| \Delta^{k} g(\vec{r})\right| d \vec{v} d \vec{r} \\
& =\left(\frac{|\lambda|}{2 \pi}\right)^{n / 2}\left\|\Delta^{k} f\right\|_{1}\left\|\Delta^{k} g\right\|_{1} .
\end{aligned}
$$

But as we have seen in Section $1, \Delta^{k} f$ and $\Delta^{k} g$ are elements of $\mathcal{S}\left(\mathbb{R}^{n}\right)$ and $\mathcal{S}\left(\mathbb{R}^{n}\right)$ is a subset of $L^{1}\left(\mathbb{R}^{n}\right)$. Hence the right hand side of the last inequality is 
a finite number and so $h(\lambda, \vec{w})$ exists for a.e. $\vec{w} \in \mathbb{R}^{n}$. Since the Paley-WienerZigmund integral $\left\langle\alpha_{j}, y\right\rangle$, for $j=1, \ldots, n$, exists for a.e. $y \in C_{0}[0, T]$, now it is easy to see that $h(\lambda,\langle\vec{\alpha}, y\rangle)$ exists for a.e. $y \in C_{0}[0, T]$.

Next we show that the convolution product of the Fourier-type functionals exists. Since we have two kinds of Fourier-type functionals, the existence theorem of the convolution product is divided into three cases. In Theorem 3.2 below, we consider the convolution product of $\Delta^{k} F$ and $\Delta^{k} G$. While in Theorem 3.3, we consider the convolution product of $\Delta^{k} F$ and $\widehat{\Delta^{k} G}$. Finally in Theorem 3.4, we consider the convolution product of $\widehat{\Delta^{k} F}$ and $\widehat{\Delta^{k} G}$.

Theorem 3.2. Let the Fourier-type functionals $\Delta^{k} F$ and $\Delta^{k} G$ are given by (9) with corresponding $f$ and $g$, respectively. Then for all nonzero real number $q$, the convolution product $\left(\Delta^{k} F * \Delta^{k} G\right)_{q}$ exists and is given by the formula

$$
\left(\Delta^{k} F * \Delta^{k} G\right)_{q}(y)=h(-i q,\langle\vec{\alpha}, y\rangle)
$$

for a.e. $y \in C_{0}[0, T]$, where $h$ is given by $(20)$.

Proof. For all $\lambda>0$, by the Wiener integration formula (11), we have

$$
\begin{aligned}
& \left(\Delta^{k} F * \Delta^{k} G\right)_{\lambda}(y) \\
= & \int_{C_{0}[0, T]} \Delta^{k} f\left(\frac{\langle\vec{\alpha}, y\rangle}{\sqrt{2}}+\frac{\langle\vec{\alpha}, x\rangle}{\sqrt{2 \lambda}}\right) \Delta^{k} g\left(\frac{\langle\vec{\alpha}, y\rangle}{\sqrt{2}}-\frac{\langle\vec{\alpha}, x\rangle}{\sqrt{2 \lambda}}\right) d m(x) \\
= & \left(\frac{\lambda}{2 \pi}\right)^{n / 2} \int_{\mathbb{R}^{n}} \Delta^{k} f\left(\frac{\langle\vec{\alpha}, y\rangle+\vec{u}}{\sqrt{2}}\right) \Delta^{k} g\left(\frac{\langle\vec{\alpha}, y\rangle-\vec{u}}{\sqrt{2}}\right) \exp \left\{-\frac{\lambda}{2}|\vec{u}|^{2}\right\} d \vec{u} \\
= & h(\lambda,\langle\vec{\alpha}, y\rangle)
\end{aligned}
$$

where $h$ is given by $(20)$. Let $\lambda \in \mathbb{C}_{+}^{\sim}$ and let $\left\{\lambda_{n}\right\}$ be a sequence in $\mathbb{C}_{+}^{\sim}$ which converges to $\lambda$. Then

$$
\begin{aligned}
& \left|\Delta^{k} f\left(\frac{\langle\vec{\alpha}, y\rangle+\vec{u}}{\sqrt{2}}\right) \Delta^{k} g\left(\frac{\langle\vec{\alpha}, y\rangle-\vec{u}}{\sqrt{2}}\right) \exp \left\{-\frac{\lambda_{n}}{2}|\vec{u}|^{2}\right\}\right| \\
\leq & \left|\Delta^{k} f\left(\frac{\langle\vec{\alpha}, y\rangle+\vec{u}}{\sqrt{2}}\right) \Delta^{k} g\left(\frac{\langle\vec{\alpha}, y\rangle-\vec{u}}{\sqrt{2}}\right) \exp \left\{-\frac{c}{2}|\vec{u}|^{2}\right\}\right|
\end{aligned}
$$

for all $n=1,2, \ldots$, where $c=\min \left\{\operatorname{Re}\left(\lambda_{n}\right): n=1,2, \ldots\right\} \geq 0$. By the same argument as in the proof of Lemma 3.1, we know that the right hand side of the above inequality is an integrable function of $\vec{u}$ on $\mathbb{R}^{n}$ for a.e. $y \in C_{0}[0, T]$. Hence we can apply dominated convergence theorem to show that

$$
\lim _{n \rightarrow \infty} h\left(\lambda_{n},\langle\vec{\alpha}, y\rangle\right)=h(\lambda,\langle\vec{\alpha}, y\rangle)
$$


and so $h(\lambda,\langle\vec{\alpha}, y\rangle)$ is a continuous function of $\lambda$ in $\mathbb{C}_{+}^{\sim}$. Let $D$ be a closed contour in $\mathbb{C}_{+}$. Then

$$
\begin{aligned}
& \int_{\mathbb{R}^{n}} \int_{D}\left|\left(\frac{\lambda}{2 \pi}\right)^{n / 2} \Delta^{k} f\left(\frac{\langle\vec{\alpha}, y\rangle+\vec{u}}{\sqrt{2}}\right) \Delta^{k} g\left(\frac{\langle\vec{\alpha}, y\rangle-\vec{u}}{\sqrt{2}}\right) \exp \left\{-\frac{\lambda}{2}|\vec{u}|^{2}\right\}\right| d \lambda d \vec{u} \\
\leq & \left(\frac{a}{2 \pi}\right)^{n / 2} \int_{\mathbb{R}^{n}} \int_{D^{\prime}}\left|\Delta^{k} f\left(\frac{\langle\vec{\alpha}, y\rangle+\vec{u}}{\sqrt{2}}\right) \Delta^{k} g\left(\frac{\langle\vec{\alpha}, y\rangle-\vec{u}}{\sqrt{2}}\right)\right| d \lambda d \vec{u} \\
= & \left(\frac{a}{2 \pi}\right)^{n / 2} l(D) \int_{\mathbb{R}^{n}}\left|\Delta^{k} f\left(\frac{\langle\vec{\alpha}, y\rangle+\vec{u}}{\sqrt{2}}\right) \Delta^{k} g\left(\frac{\langle\vec{\alpha}, y\rangle-\vec{u}}{\sqrt{2}}\right)\right| d \vec{u},
\end{aligned}
$$

where $a=\max \{|\lambda|: \lambda \in D\}$ and $l(D)$ denotes the length of the contour $D$. By the same argument as in the proof of Lemma 3.1, we know that the integral on the right hand side of the last inequality is finite for a.e. $y \in C_{0}[0, T]$. Hence we can apply Fubini theorem to show that

$$
\begin{aligned}
& \int_{D} h(\lambda,\langle\vec{\alpha}, y\rangle) d \lambda \\
= & \int_{\mathbb{R}^{n}} \int_{D}\left(\frac{\lambda}{2 \pi}\right)^{n / 2} \Delta^{k} f\left(\frac{\langle\vec{\alpha}, y\rangle+\vec{u}}{\sqrt{2}}\right) \Delta^{k} g\left(\frac{\langle\vec{\alpha}, y\rangle-\vec{u}}{\sqrt{2}}\right) \exp \left\{-\frac{\lambda}{2}|\vec{u}|^{2}\right\} d \lambda d \vec{u} .
\end{aligned}
$$

But since the integrand on the right hand side of the above equation is an analytic function of $\lambda$ in $\mathbb{C}_{+}$, the inner integral is equal to 0 . Hence by the Morera's theorem, $h(\lambda,\langle\vec{\alpha}, y\rangle)$ is an analytic function of $\lambda$ in $\mathbb{C}_{+}$. Finally, we can apply dominated convergence theorem once more to show that

$$
\left(\Delta^{k} F * \Delta^{k} G\right)_{q}(y)=\lim _{\lambda \rightarrow-i q} h(\lambda,\langle\vec{\alpha}, y\rangle)=h(-i q,\langle\vec{\alpha}, y\rangle)
$$

for a.e. $y \in C_{0}[0, T]$ as we wished to prove.

A careful look at the proofs of Lemma 3.1 and Theorem 3.2, we see that the essential conditions to ensure the results are the integrability of $\Delta^{k} f$ and $\Delta^{k} g$. Since $\widehat{\Delta^{k} f}$ and $\widehat{\Delta^{k} g}$ are also elements of $L^{1}\left(\mathbb{R}^{n}\right)$, we have the following theorems. We just state them without proofs.

Theorem 3.3. Let the Fourier-type functionals $\Delta^{k} F$ and $\widehat{\Delta^{k} G}$ are given by (9) and (10) with corresponding $f$ and $g$ in $\mathcal{S}\left(\mathbb{R}^{n}\right)$, respectively. Then for all nonzero real number $q$, the convolution product $\left(\Delta^{k} F * \widehat{\Delta^{k} G}\right)_{q}$ exists and is given by the formula

$$
\begin{aligned}
& \left(\Delta^{k} F * \widehat{\Delta^{k} G}\right)_{q}(y) \\
= & \left(\frac{\lambda}{2 \pi}\right)^{n / 2} \int_{\mathbb{R}^{n}} \Delta^{k} f\left(\frac{\langle\vec{\alpha}, y\rangle+\vec{u}}{\sqrt{2}}\right) \widehat{\Delta^{k}} g\left(\frac{\langle\vec{\alpha}, y\rangle-\vec{u}}{\sqrt{2}}\right) \exp \left\{-\frac{\lambda}{2}|\vec{u}|^{2}\right\} d \vec{u},
\end{aligned}
$$

for a.e. $y \in C_{0}[0, T]$.

As we noted in Section 1, the convolution product for the Fourier-Feynman transform is commutative. Hence we know that $\left(\widehat{\Delta^{k} F} * \Delta^{k} G\right)_{q}$ also exists. 
Theorem 3.4. Let the Fourier-type functionals $\widehat{\Delta^{k} F}$ and $\widehat{\Delta^{k} G}$ are given by (10) with corresponding $f$ and $g$ in $\mathcal{S}\left(\mathbb{R}^{n}\right)$, respectively. Then for all nonzero real number $q$, the convolution product $\left(\widehat{\Delta^{k} F} * \widehat{\Delta^{k} G}\right)_{q}$ exists and is given by the formula

$$
\begin{aligned}
& \left(\widehat{\Delta^{k} F} * \widehat{\Delta^{k} G}\right)_{q}(y) \\
= & \left(\frac{\lambda}{2 \pi}\right)^{n / 2} \int_{\mathbb{R}^{n}} \widehat{\Delta^{k} f}\left(\frac{\langle\vec{\alpha}, y\rangle+\vec{u}}{\sqrt{2}}\right) \widehat{\Delta^{k}} g\left(\frac{\langle\vec{\alpha}, y\rangle-\vec{u}}{\sqrt{2}}\right) \exp \left\{-\frac{\lambda}{2}|\vec{u}|^{2}\right\} d \vec{u},
\end{aligned}
$$

for a.e. $y \in C_{0}[0, T]$.

Although the right hand sides of (22) and (23) can be expressed further using the equations (8) for the Fourier transform $\widehat{\Delta^{k} F}$ and $\widehat{\Delta^{k} G}$, we will not give them here.

In our next theorem we show that the Fourier Feynman transform of convolution product of Fourier-type functionals $\Delta^{k} F$ and $\Delta^{k} G$ is the product of transforms of each functionals.

Theorem 3.5. Let the Fourier-type functionals $\Delta^{k} F$ and $\Delta^{k} G$ are given by (9) with corresponding $f$ and $g$ in $\mathcal{S}\left(\mathbb{R}^{n}\right)$, respectively. Let $1 \leq p<\infty$ and let $q$ be a nonzero real number. Then

$$
T_{q}^{(p)}\left(\left(\Delta^{k} F * \Delta^{k} G\right)_{q}\right)(y)=T_{q}^{(p)}\left(\Delta^{k} F\right)\left(\frac{y}{\sqrt{2}}\right) T_{q}^{(p)}\left(\Delta^{k} G\right)\left(\frac{y}{\sqrt{2}}\right),
$$

for a.e. $y \in C_{0}[0, T]$.

Proof. For all $\lambda>0$, by the expression (21) for convolution product of $\Delta^{k} F$ and $\Delta^{k} G$, we have

$$
\begin{aligned}
T_{\lambda}\left(\left(\Delta^{k} F * \Delta^{k} G\right)_{q}\right)(y) & =\int_{C_{0}[0, T]}\left(\Delta^{k} F * \Delta^{k} G\right)_{q}\left(\lambda^{-1 / 2} x+y\right) d m(x) \\
& =\int_{C_{0}[0, T]} h\left(-i q, \lambda^{-1 / 2}\langle\vec{\alpha}, x\rangle+\langle\vec{\alpha}, y\rangle\right) d m(x) .
\end{aligned}
$$

By the Wiener integration formula (11) and the expression (20) for $h$, we have

$$
\begin{aligned}
& T_{\lambda}\left(\left(\Delta^{k} F * \Delta^{k} G\right)_{q}\right)(y) \\
= & \left(\frac{\lambda}{2 \pi}\right)^{n / 2} \int_{\mathbb{R}^{n}} h(-i q, \vec{v}+\langle\vec{\alpha}, y\rangle) \exp \left\{-\frac{\lambda}{2}|\vec{v}|^{2}\right\} d \vec{v} \\
= & \left(\frac{\lambda}{2 \pi}\right)^{n / 2}\left(\frac{-i q}{2 \pi}\right)^{n / 2} \int_{\mathbb{R}^{n}} \int_{\mathbb{R}^{n}} \Delta^{k} f\left(\frac{\vec{v}+\vec{u}+\langle\vec{\alpha}, y\rangle}{\sqrt{2}}\right) \Delta^{k} g\left(\frac{\vec{v}-\vec{u}+\langle\vec{\alpha}, y\rangle}{\sqrt{2}}\right) \\
& \exp \left\{-\frac{\lambda}{2}\left(|\vec{v}|^{2}+|\vec{u}|^{2}\right)\right\} d \vec{u} d \vec{v} .
\end{aligned}
$$


Letting $\vec{w}=(\vec{v}+\vec{u}) / \sqrt{2}$ and $\vec{r}=(\vec{v}-\vec{u}) / \sqrt{2}$, we have

$$
\begin{aligned}
& T_{\lambda}\left(\left(\Delta^{k} F * \Delta^{k} G\right)_{q}\right)(y) \\
= & \left(\frac{\lambda}{2 \pi}\right)^{n / 2}\left(\frac{-i q}{2 \pi}\right)^{n / 2}\left(\int_{\mathbb{R}^{n}} \Delta^{k} f(\vec{w}) \exp \left\{-\frac{\lambda}{2}\left|\vec{w}-\frac{\langle\vec{\alpha}, y\rangle}{\sqrt{2}}\right|^{2}\right\} d \vec{w}\right) \\
& \left(\int_{\mathbb{R}^{n}} \Delta^{k} g(\vec{r}) \exp \left\{-\frac{\lambda}{2}\left|\vec{r}-\frac{\langle\vec{\alpha}, y\rangle}{\sqrt{2}}\right|^{2}\right\} d \vec{r}\right) .
\end{aligned}
$$

Now by the same argument as in the proof of Theorem 2.1, we can show that the last expression is analytic in $\lambda \in \mathbb{C}_{+}^{\sim}$ and we have

$$
\begin{aligned}
T_{q}^{(p)}\left(\left(\Delta^{k} F * \Delta^{k} G\right)_{q}\right)(y)= & \left(\frac{-i q}{2 \pi}\right)^{n}\left(\int_{\mathbb{R}^{n}} \Delta^{k} f(\vec{w}) \exp \left\{\frac{i q}{2}\left|\vec{w}-\frac{\langle\vec{\alpha}, y\rangle}{\sqrt{2}}\right|^{2}\right\} d \vec{w}\right) \\
& \left(\int_{\mathbb{R}^{n}} \Delta^{k} g(\vec{r}) \exp \left\{\frac{i q}{2}\left|\vec{r}-\frac{\langle\vec{\alpha}, y\rangle}{\sqrt{2}}\right|^{2}\right\} d \vec{r}\right) .
\end{aligned}
$$

Finally by Theorem 2.1 we complete the proof.

Considering Theorems 3.3, 3.4 and the proof of Theorem 3.5, we see that the relationship (24) holds for the Fourier-type functionals $\widehat{\Delta^{k} F}$ and $\widehat{\Delta^{k} G}$. We state the results in Theorems 3.6 and 3.7 below without proofs.

Theorem 3.6. Let the Fourier-type functionals $\Delta^{k} F$ and $\widehat{\Delta^{k} G}$ are given by (9) and (10) with corresponding $f$ and $g$ in $\mathcal{S}\left(\mathbb{R}^{n}\right)$, respectively. Let $1 \leq p<\infty$ and let $q$ be a nonzero real number. Then

$$
T_{q}^{(p)}\left(\left(\Delta^{k} F * \widehat{\Delta^{k} G}\right)_{q}\right)(y)=T_{q}^{(p)}\left(\Delta^{k} F\right)\left(\frac{y}{\sqrt{2}}\right) T_{q}^{(p)}\left(\widehat{\Delta^{k} G}\right)\left(\frac{y}{\sqrt{2}}\right)
$$

for a.e. $y \in C_{0}[0, T]$.

Theorem 3.7. Let the Fourier-type functionals $\widehat{\Delta^{k} F}$ and $\widehat{\Delta^{k} G}$ are given by (10) with corresponding $f$ and $g$ in $\mathcal{S}\left(\mathbb{R}^{n}\right)$, respectively. Let $1 \leq p<\infty$ and let $q$ be a nonzero real number. Then

$$
T_{q}^{(p)}\left(\left(\widehat{\Delta^{k} F} * \widehat{\Delta^{k} G}\right)_{q}\right)(y)=T_{q}^{(p)}\left(\widehat{\Delta^{k} F}\right)\left(\frac{y}{\sqrt{2}}\right) T_{q}^{(p)}\left(\widehat{\Delta^{k} G}\right)\left(\frac{y}{\sqrt{2}}\right)
$$

for a.e. $y \in C_{0}[0, T]$.

\section{References}

[1] J.M. Ahn, K.S. Chang, B.S. Kim and I. Yoo, Fourier-Feynman transform, convolution and first variation, Acta Math. Hungar. 100 (2003), 215-235.

[2] R.H. Cameron and D.A. Storvick, An $L_{2}$ analytic Fourier-Feynman transform, Michigan Math. J. 23 (1976), 1-30.

[3] K.S. Chang, D.H. Cho, B.S. Kim, T.S. Song and I. Yoo, Relationships involving generalized Fourier-Feynman transform, convolution and first variation, Integral Transforms Spec. Funct. 16 (2005), 391-405.

[4] K.S. Chang, B.S. Kim and I. Yoo, Fourier-Feynman transform, convolution and first variation of functionals on abstract Wiener space, Integral Transforms Spec. Funct. 10 (2000), 179-200. 
[5] _ Analytic Fourier-Feynman transform and convolution of functionals on abstract Wiener space, Rocky Mountain J. Math. 30 (2000), 823-842.

[6] H.S. Chung and V.K. Tuan, Fourier-type functionals on Wiener space, Bull. Korean Math. Soc. 49 (2012), 609-619.

[7] T. Huffman, C. Park and D. Skoug, Analytic Fourier-Feynman transforms and convolution, Trans. Amer. Math. Soc. 347 (1995), 661-673.

[8] Convolutions and Fourier-Feynman transforms of functionals involving multiple integrals, Michigan Math. J. 43 (1996), 247-261.

[9] G.W. Johnson and D.L. Skoug, An $L_{p}$ analytic Fourier-Feynman transform, Michigan Math. J. 26 (1979), 103-127.

[10] B.J. Kim and B.S. Kim, Relationships among Fourier-Yeh-Feynman transform, convolution and the first variation on Yeh-Wiener space, Honam Math. J. 33 (2011), 207-221.

[11] B.S. Kim and Y.K. Yang, Fourier-Yeh-Feynman transform and convolution on YehWiener space, Korean J. Math. 16 (2008), 335-348.

[12] C. Park, D. Skoug and D. Storvick, Relationships among the first variation, the convolution product, and the Fourier-Feynman transform, Rocky Mountain J. Math. 28 (1998), 1447-1468.

[13] D. Skoug and D. Storvick, A survey of results involving transforms and convolutions in function space, Rocky Mountain J. Math. 34 (2004), 1147-1175.

[14] J. Yeh, Convolution in Fourier-Wiener transform, Pacific J. Math. 15 (1965), 731-738.

[15] I. Yoo, Convolution and the Fourier-Wiener transform on abstract Wiener space, Rocky Mountain J. Math. 25 (1995), 1577-1587.

School of Liberal Arts, Seoul National University of Science and Technology, SEOUl 139-743, Korea

E-mail address: mathkbs@seoultech.ac.kr 